\title{
Efficiency of the Stool-PCR Test Targeting NADH Dehydrogenase (Nad) Subunits for Detection of Opisthorchis viverrini Eggs
}

\author{
Wansika Phadungsil (D, ${ }^{1}$ Supaporn Pumpa, ${ }^{2}$ Kridsada Sirisabhabhorn, ${ }^{2}$ \\ Amornrat Geadkaew-Krenc $\mathbb{D}^{1},{ }^{1}$ Rudi Grams $\left(\mathbb{D},{ }^{1}\right.$ Mathirut Mungthin, ${ }^{3}$ \\ Toon Ruang-Areerate, ${ }^{4}$ Poom Adisakwattana ${ }^{(D},{ }^{5}$ Nipawan Labbunruang, 6 \\ and Pongsakorn Martviset $\mathbb{B D}^{7}$
}

\author{
${ }^{1}$ Graduate Program in Biomedical Sciences, Faculty of Allied Health Sciences, Thammasat University, Pathumthani 12120, \\ Thailand \\ ${ }^{2}$ Department of Medical Technology Laboratory, Thammasat University Hospital, Pathumthani 12120, Thailand \\ ${ }^{3}$ Department of Phamacology, Phramongkutklao College of Medicine, Bangkok 10400, Thailand \\ ${ }^{4}$ Department of Parasitology, Phramongkutklao College of Medicine, Bangkok 10400, Thailand \\ ${ }^{5}$ Department of Helminthology, Faculty of Tropical Medicine, Mahidol University, Bangkok 10400, Thailand \\ ${ }^{6}$ Department of Biology, Faculty of Science, Udon Thani Rajabhat University, Udon Thani 41000, Thailand \\ ${ }^{7}$ Division of Parasitology, Department of Preclinical Science, Faculty of Medicine, Thammasat University, Pathumthani 12120, \\ Thailand
}

Correspondence should be addressed to Pongsakorn Martviset; pong_m@tu.ac.th

Received 25 October 2021; Revised 8 November 2021; Accepted 26 November 2021; Published 6 December 2021

Academic Editor: Pedro P. Chieffi

Copyright (C) 2021 Wansika Phadungsil et al. This is an open access article distributed under the Creative Commons Attribution License, which permits unrestricted use, distribution, and reproduction in any medium, provided the original work is properly cited.

Opisthorchis viverrini infection is the major parasitic infection problem in Southeast Asian countries, and long-term infection will lead to cholangiocarcinoma (CCA), the bile duct cancer. The early diagnosis of $O$. viverrini infection may interrupt the progression of the opisthorchiasis and other related illnesses, especially CCA. The current diagnostic procedure is stool examination by microscope-based methods such as direct smear and concentration techniques but it is limited by low parasite egg numbers. The molecular diagnosis prompts the chance to evaluate the light infection with low number of parasite eggs but is currently inconvenient for routine use due to special equipment requirement and unstable sensitivities. Our present study aims to establish the efficiency of $\mathrm{O} v \mathrm{Nad}$ subunits, the mitochondrial gene, for introducing as a potential diagnostic target by conventional PCR, the cheapest and easiest molecular procedure. A total of 166 stool samples were investigated microscopically by the PBS-ethyl acetate concentration technique (PECT); 75 samples were O. viverrini positive with 28 samples that were positive with single parasite (hookworm, A. lumbricoides, S. stercoralis, Taenia spp., and T. trichiura), 11 samples were with mixed infection, and 52 samples were without parasite detection. The detection limits of $\mathrm{O} v \mathrm{Nad}$ subunits were evaluated in artificially spiked samples containing 0 , $1,5,10,20,50$, and $100 \mathrm{O} v$-eggs. The result suggested that the best detection efficacy was of $\mathrm{O} v \mathrm{Nad} 5$ that had exact detection limits at only 5 eggs. In the PCR amplification of OvNad subunits, there exist $100 \%$ specificities with varied sensitivities from $64 \%, 88 \%$, $80 \%$, and $100 \%$ of $\mathrm{O} v \mathrm{Nad} 1, \mathrm{O} v \mathrm{Nad} 2, \mathrm{O} v \mathrm{Nad} 4$, and $\mathrm{O} v \mathrm{Nad}$, respectively. OvNad subunits were amplified specifically without cross reactivity with the other collected parasites. Our study established that OvNad subunits, especially OvNad5, are the potent candidates for PCR amplification of stool containing $O v$-eggs with high confidential sensitivity, specificity, PPV, and NPV even in the light infection that would be a benefit for developing as a routine diagnosis of O. viverrini infection. 


\section{Introduction}

Opisthorchis viverrini (O. viverrini; Ov), a digenean liver fluke, is a major cause of human opisthorchiasis and $O v$ induced cholangiocarcinoma (CCA), the biliary ductal cancer, in enduring infection [1]. It is a food-borne parasite that spreads throughout the Southeast Asian subregional countries, especially in the Mekong Basin including Laos PDR, Cambodia, Vietnam, and Thailand [2-4]. Huge incidence of $O$. viverrini is highly related to the prevalence of CCA in those areas according to the consumption of raw Cyprinid fish, a secondary intermediate host of $O$. viverrini [5]. CCA is a highly progressive cancer originating from the transformed intra- or extrahepatic cholangiocytes which lack early diagnostic markers [6]. The diagnosis of $O$. viverrini infection is possible, but it has a lot of limitations. The gold standard for diagnosis of $O$. viverrini infection is microscope-based methods such as direct smear and concentration techniques including the formalin-ethyl acetate concentration technique (FECT) and related procedures [7]. The conventional methods are convenient but have low sensitivity; moreover, the observation could be erroneous and need professional laboratorians for identification by their naked eyes [8].

The molecular detection of $\mathrm{Ov}$-eggs has been introduced for several years by many methods such as conventional PCR, LAMP, and quantitative real-time PCR [9-14]. The target genes are varied but mostly concentrated on the mitochondrial gene due to the presence of high conservation such as internal transcribed spacer (ITS), cytochrome c oxidase 1 (cox1), and NADH dehydrogenase 1 (nad1) [15-18]. The detection limit of those genes is not stable among the specimen types, collection procedures, preservation methods, extraction protocols, and the number of eggs [19].

$\mathrm{NADH}$ dehydrogenase (Nad) is the enzyme which converts nicotinamide adenine dinucleotide (NAD) from $\mathrm{NADH}$ (reduced form) to $\mathrm{NAD}^{+}$(oxidized form) encoded by the mitochondrial genome [20]. The NADH dehydrogenase complex spans inside the inner membrane of the mitochondria. The complex provides energy from the oxidation of $\mathrm{NADH}$ to $\mathrm{NAD}^{+}$and proton transfer from the mitochondrial matrix to the intermembrane space. Moreover, the complex has numerous metabolic functions such as fatty acid oxidation, TCA cycle, amino acid catabolism, and cytosolic reactions given the crucial central position of metabolic pathways responsible for carbohydrate, fatty acid, and amino acid catabolism [21]. As it has various vital functions for the cells, Nad is abundantly expressed in every cell which is a good compelling target for molecular detection. The established mitochondrial genome of $O$. viverrini permits the possibility to encounter the Nad subunits for development as the diagnostic target other than Nad1 that was previously tested [17]. The schematic drawing of the $O$. viverrini mitochondrial genome is illustrated in Figure 1. Hence, this study aimed to establish the new target for the molecular identification of $\mathrm{Ov}$-egg directly in the stool specimen by comparison of the efficiency of $\mathrm{Nad}$ subunits using conventional PCR which is the easiest and cheapest molecular procedure for developing a potential diagnostic target in the future.

\section{Materials and Methods}

2.1. Study Areas and Stool Collection. A total of 166 stool samples were collected from several provinces in the north, northeast, and central Thailand where O. viverrini is endemic in Chiang Mai, Phrae, Phetchabun, Nakhon Phanom, Pathumthani, and Chachoengsao provinces during 2018 to 2020. The participants living in the risk areas of these provinces were included in this study. All participants were educated and consented for a noninvasive stool collection procedure. The collected samples were transferred to the laboratory and kept in $4-10^{\circ} \mathrm{C}$ until processed by the concentration method.

\subsection{PBS-Ethyl Acetate Concentration Technique (PECT).} All samples were subjected to the PBS-ethyl acetate concentration technique (PECT) as previously described [15] with a few modifications. In brief, one gram of each stool specimen was mixed with $10 \mathrm{~mL}$ of PBS, $\mathrm{pH} 7.4$, and filtered through three layers of gauze pad. $3 \mathrm{~mL}$ of ethyl acetate was added to the filtrate and mixed thoroughly by inverting. The tube was centrifuged at $2,000 \times g$, for $10 \mathrm{~min}$, and the upper phases were removed. The sediment was investigated under the light microscope at 100 and 400 magnifications.

2.3. Genomic DNA Isolation. The genomic DNA of parasitic eggs was isolated from PECT sediments of stool. Before extraction, the sediments were autoclaved at $121^{\circ} \mathrm{C}$, for $5 \mathrm{~min}$ to open the operculum of parasite eggs. The genomic DNAs were then extracted by using the QIAamp ${ }^{\circledR}$ PowerFecal $^{\circledR}$ DNA kit (QIAGEN, Germany) according to the manufacturer's instructions with the final filtrates containing $50 \mu \mathrm{L}$ of eluted DNAs.

2.4. Primer Design and PCR Amplification. OvNad subunit specific primers for PCR amplification were designed from a partial sequence of O. viverrini mitochondrial DNA available in GenBank (accession number JF739555.1). The primer sequences of Ovnad1, Ovnad2, Ovnad4, and Ovnad5 are presented in Table 1. The expected amplicons of Ovnad1, Ovnad2, Ovnad4, and Ovnad5 were 204 bp, 198 bp, 200 bp, and $199 \mathrm{bp}$, respectively. PCR amplifications were performed by using GoTaq ${ }^{\circledR}$ Colorless Master Mix (Promega, USA) containing $3 \mu \mathrm{L}$ of genomic DNA and 25 pmole of each forward and reverse primer in a thermal cycler (Mastercycler nexus Eppendorf flexlid, Germany). The amplification steps included initial denaturation at $95^{\circ} \mathrm{C}$ for $5 \mathrm{~min}$, followed by 35 cycles of denaturation at $95^{\circ} \mathrm{C}$ for $1 \mathrm{~min}$, annealing at $55^{\circ} \mathrm{C}$ for $1 \mathrm{~min}$, extension at $72^{\circ} \mathrm{C}$ for $1 \mathrm{~min}$, and one cycle of a final extension at $72^{\circ} \mathrm{C}$ for $10 \mathrm{~min}$. The PCR products were size separated on $2 \%$ agarose gel containing ViSafe Red Gel Stain (Vivantis, USA) using 1X TBE buffer at $100 \mathrm{~V}$ for $2 \mathrm{~h}$. The PCR products were 


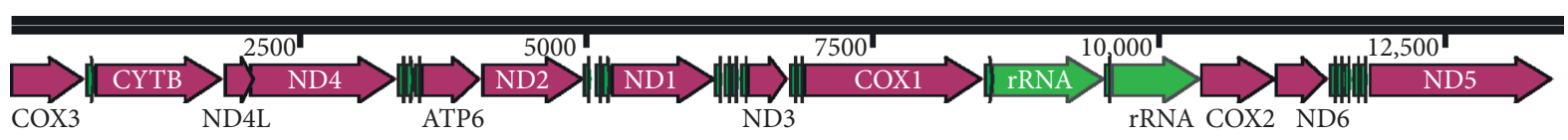

Figure 1: Schematic drawing of the O. viverrini mitochondrial genome showing the composition of Nad subunits (ND).

TABLE 1: Sequences of primer sets for amplification of OvNad subunits.

\begin{tabular}{lc}
\hline Primer & Sequences (from $5^{\prime}$ to $3^{\prime}$ ) \\
\hline OvNad $1(\mathrm{~F})$ & GATTACGCAGAAGCGGAGAG \\
OvNad2 (F) & TAGCTCAAGGGCTTCTTTGG \\
OvNad4 (F) & GGCTTCCAATGTTGCTCTGT \\
OvNad5 (F) & TTTGCGGAGGTTTGTTACCT \\
OvNad $1(\mathrm{R})$ & AAGAGTAGCACGAGCCCAGA \\
O $v N a d 2(\mathrm{R})$ & ACTGCTACTGAACCCGGAAA \\
O $v N a d 4(\mathrm{R})$ & TACCGAAACAGGCCTACTGG \\
OvNad5 (R) & CACCTCACCAATTCAACACG \\
\hline
\end{tabular}

confirmed for their conformation by DNA sequencing service (Macrogen, Republic of Korea).

\subsection{Detection Limit Determination of OvNad Subunits.}

The detection limits of $\mathrm{O} v \mathrm{Nad}$ subunits were evaluated by using various numbers of $O$. viverrini eggs from adult parasites obtained from the livers of infected hamsters (the animal ethics was approved by the Thammasat Animal Care and Use Committee (TU-ACUC), no. 024/2559, following the care and use of laboratory animals' guideline (NRC 2011). The O. viverrini eggs of $0,1,5,10,20,50$, and 100 were artificially inoculated with $250 \mathrm{mg}$ of $O$. viverrini-negative stools and processed for genomic DNA isolation. PCR amplifications were performed by using GoTaq ${ }^{\circledR}$ Colorless Master Mix (Promega, USA) containing $3 \mu \mathrm{L}$ of genomic DNA and 25 pmole of each forward and reverse primer in a thermal cycler (Mastercycler nexus Eppendorf flexlid, Germany). The amplification steps included initial denaturation at $95^{\circ} \mathrm{C}$ for $5 \mathrm{~min}$, followed by 35 cycles of denaturation at $95^{\circ} \mathrm{C}$ for $1 \mathrm{~min}$, annealing at $55^{\circ} \mathrm{C}$ for $1 \mathrm{~min}$, extension at $72^{\circ} \mathrm{C}$ for $1 \mathrm{~min}$, and one cycle of a final extension at $72^{\circ} \mathrm{C}$ for $10 \mathrm{~min}$. The PCR products were size separated on $2 \%$ agarose gel containing ViSafe Red Gel Stain (Vivantis, USA) using $1 \mathrm{X}$ TBE buffer at $100 \mathrm{~V}$ for $2 \mathrm{~h}$. The PCR products were confirmed for their correct sequences by DNA sequencing service (Macrogen, Republic of Korea).

2.6. Cross-Reactivity Evaluation. The cross reactivities of OvNad subunit specific primers were simultaneously investigated in the collected stools. The PECT sediments with other parasites were monitored for the cross reactivity. The samples included both single and mixed infections with either flatworms or roundworms.

2.7. Statistical Analysis. The sensitivity, specificity, positive predicted value (PPV), and negative predicted value (NVP) of OvNad subunit detection by PCR in the stool were calculated as previously described [22] comparing with PECT as a gold standard method.

\section{Result}

3.1. Parasite Detected by the Modified PBS-Ethyl Acetate Concentration Technique (PECT). From 166 stool samples, the PECT result revealed the positive samples including $O$. viverrini (75/166), hookworm (11/166), Trichuris trichiura (6/166), Strongyloides stercoralis $(5 / 166)$, Taenia spp. (3/ 166), and Ascaris lumbricoides (3/166). Moreover, the mixed infection has also been identified which comprised hookworm $+A$. lumbricoides (5/166), hookworm $+T$. trichiura (1/166), A. lumbricoides + Enterobius vermicularis (2/ 166), A. lumbricoides $+T$. trichiura (1/166), and Taenia spp. + E. vermicularis $(2 / 166)$. The rest of the samples were negative for PECT (52/166). The result is shown in Table 2.

3.2. Detection Limits of OvNad Subunits. The PCR amplification of $\mathrm{OvNad}$ subunits by conventional PCR with $0,1,5$, $10,20,50$, and $100 \mathrm{Ov}$-egg reviewed the different detection limits among the $\mathrm{O} v \mathrm{Nad}$ subunits. OvNad1 has the lowest detection limit at 50 eggs followed by $\mathrm{OvNad} 4$ at 20 eggs and $\mathrm{O} v \mathrm{Nad} 2$ at 10 eggs. Interestingly, $\mathrm{O} v \mathrm{Nad} 5$ had unyielding activities in the low egg number in only 5 eggs. The amplification of $\mathrm{OvNad}$ subunits is illustrated in Figure 2.

3.3. Sensitivity, Specificity, and Cross Reactivity of OvNad Subunits. The PCR amplification of 166 samples with $\mathrm{O} v \mathrm{Nad}$ subunit specific primers suggested that $\mathrm{O} v \mathrm{Nad}$ subunits had different sensitivities. Only 48/75 of PECTpositive samples can be amplified by OvNad1, while 66/75 and $60 / 75$ were successfully amplified by $\mathrm{OvNad} 2$ and $\mathrm{O} v \mathrm{Nad} 4$, respectively. The OvNad5 showed luminous activity with 75/75 of PECT-positive samples and another 2 from PECT-negative samples (the sequences had been correctly confirmed). The sensitivities of OvNad1, OvNad2, $\mathrm{O} v \mathrm{Nad} 4$, and $\mathrm{O} v \mathrm{Nad} 5$ were $64.0 \%, 88.0 \%, 80.0 \%$, and $100 \%$, respectively, and specificities were $100 \%$ in all subunits. The positive predicted values (PPV) of OvNad1, OvNad2, $\mathrm{O} v \mathrm{Nad} 4$, and $\mathrm{OvNad} 5$ were $100 \%$ in all subunits, whereas the negative predicted values (NVP) were $77.12 \%, 91.00 \%$, $85.85 \%$, and $100 \%$, respectively. The sensitivities, specificities, PPV, and NPV are shown in Table 3. In the testing of cross reactivities, the $\mathrm{O} v \mathrm{Nad}$ subunits were used to amplify in all simultaneously collected parasites of both single and mixed infections. All $\mathrm{O} v \mathrm{Nad}$ subunits showed excellency without amplification of all other kinds of parasites as mentioned in Table 2 and Figure 3.

\section{Discussion}

The molecular technology allows many researchers to develop several diagnostic procedures for detecting of any diseases, especially infectious diseases with perilous 
TABle 2: The number of parasite-positive samples by PECT and PCR amplification of OvNad subunits.

\begin{tabular}{lcccc}
\hline \multirow{2}{*}{ Parasites } & \multirow{2}{*}{ Positive samples by PECT } & \multicolumn{3}{c}{ Positive samples by PCR amplification of } \\
& & OvNad1 & OvNad2 & OvNad4 \\
\hline O. viverrini & 75 & 48 & 66 & 60 \\
Hookworm & 11 & 0 & 0 & 0 \\
A. lumbricoides & 3 & 0 & 0 & 0 \\
Taenia spp. & 3 & 0 & 0 & 0 \\
S. stercoralis & 5 & 0 & 0 & 0 \\
T. trichiura & 6 & 0 & 0 & 0 \\
Hookworm + A. lumbricoides & 5 & 0 & 0 & 0 \\
Hookworm + T. trichiura & 1 & 0 & 0 & 0 \\
A. lumbricoides + E. vermicularis & 2 & 0 & 0 & 0 \\
A. lumbricoides + T. trichiura & 1 & 0 & 0 & 0 \\
Taenia spp + E. vermicularis & 2 & 0 & 0 & 0 \\
No parasite detection & 52 & 0 & 0 & 0 \\
Total & 166 & 48 & 0 & 0 \\
\hline
\end{tabular}
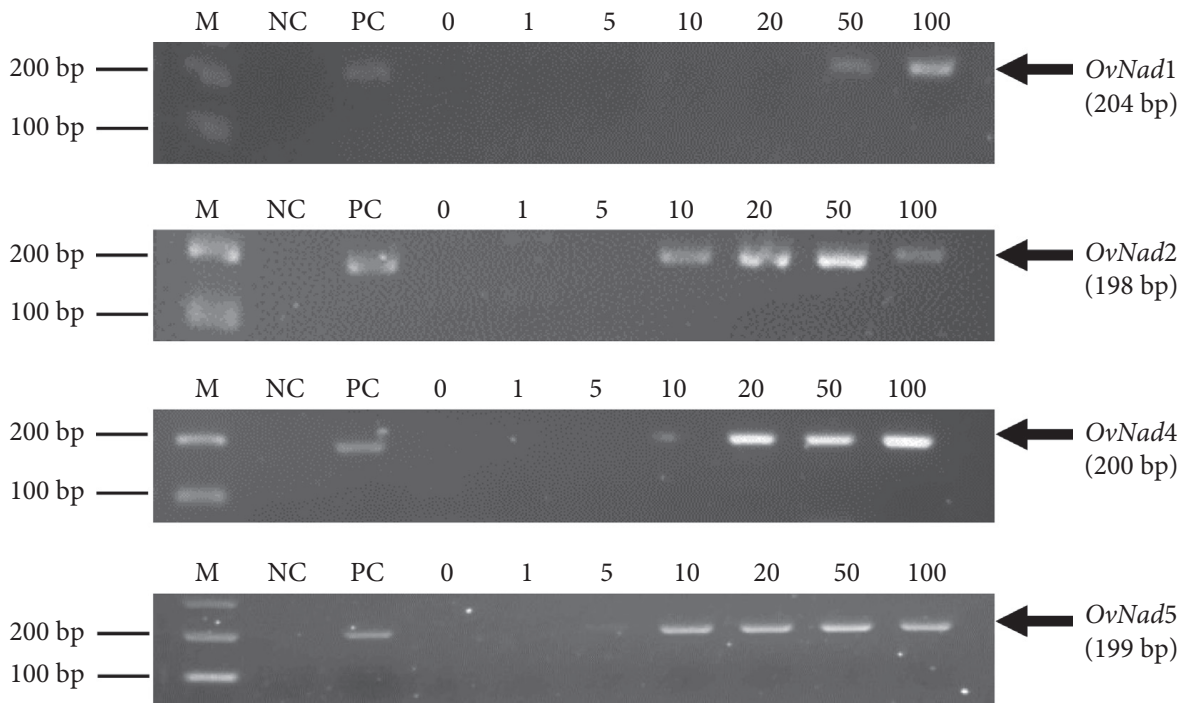

Figure 2: The detection limits of $\mathrm{O} v \mathrm{Nad}$ subunits in various numbers of eggs in artificially spiked samples (M, 100 bp DNA marker; NC, no template control; PC, positive control from uncountable $O v$-eggs dissected from adult $O$. viverrini; and 0, 1, 5, 10, 20, 50, and 100 are the number of $\mathrm{O} v$-eggs inoculated in stool specimens).

TABLE 3: Sensitivity, specificity, positive predicted value (PPV), and negative predicted value (NPV) of OvNad subunit detection by PCR amplification compared with PECT.

\begin{tabular}{lcccc}
\hline OvNad subunits & Sensitivity (\%) & Specificity (\%) & Positive predicted value (PPV) & Negative predicted value (NVP) \\
\hline OvNad 1 & 64.00 & 100.00 & 100.00 & 77.12 \\
OvNad2 & 88.00 & 100.00 & 100.00 & 91.00 \\
OvNad4 & 80.00 & 100.00 & 100.00 & 85.85 \\
OvNad5 & 100.00 & 100.00 & 100.00 & 100.00 \\
\hline
\end{tabular}

complexity. The $O$. viverrini infection is also in the same pipeline with other diseases, which has some of established molecular diagnostic procedures, but the sensitivity has been limited. The ITS-based PCR is the first and most famously used target for molecular identification, but later, it showed varied sensitivity comparable with the newly identified targets such as Cox1, Cytochrome B (CytB), and Nad1 [17]. Even though these targets showed better or equal sensitivities to ITS, they have many drawbacks, especially the detection in the low number of parasite eggs [10]. Nad1 is a mitochondrial gene which has been previously introduced but never verified with the other Nad subunits. The detection limits of OvNad subunits were verified in our study. The result revealed that the $O v N a d 5$ subunit had better activities than the other subunits. Only 5 eggs could be detected by OvNad5 primer sets. When compared with the other established gene targets, also OvNad subunits, especially $\mathrm{O} v \mathrm{Nad}$, have better or equal capabilities to detect the lower concentration of eggs (5 EPG in artificially spiked samples) to ITS-2 and Cox1 [10, 11, 23, 24]. 


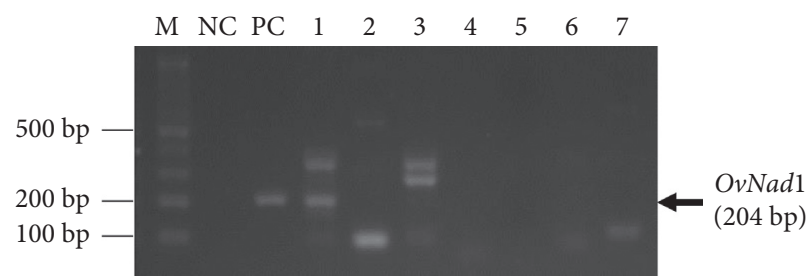

(a)

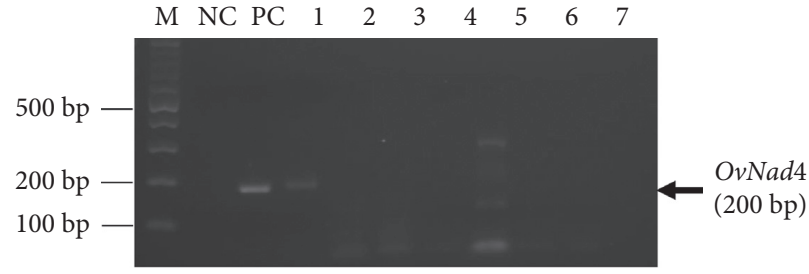

(c)

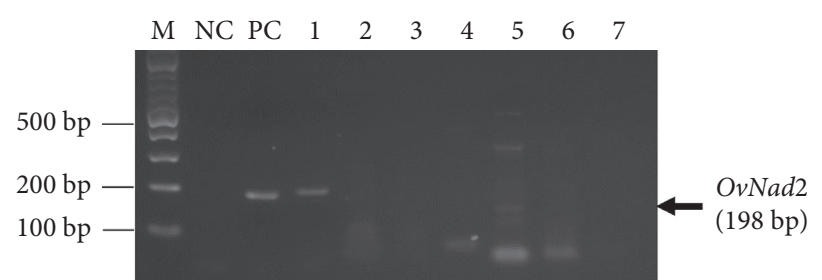

(b)

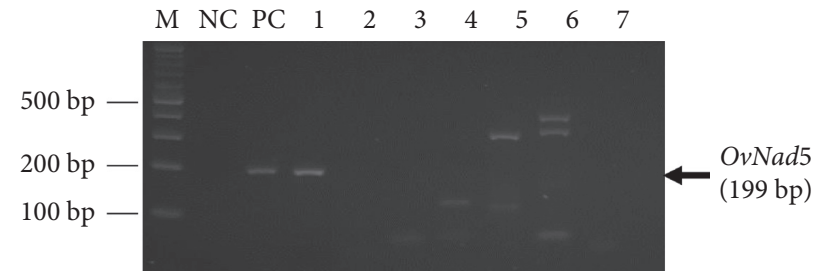

(d)

FIGURE 3: PCR amplicons of $\mathrm{O} v \mathrm{Nad}$ subunits using the new introduced primers and its cross-reactivity evaluation. (a) $\mathrm{O} v \mathrm{Nad} 1$, (b) $\mathrm{O} v \mathrm{Nad} 2$, (c) OvNad4, and (d) OvNad5 (M, 100 bp plus DNA marker; NC, no template control, PC; positive control from uncountable Ov-eggs dissected from adult $O$. viverrini; and 1 to 7 are the samples processed by PECT, 1 , O. viverrini egg detected sample; 2, parasite undetected sample; 3, hookworm egg detected sample; 4, Ascaris lumbricoides egg detected sample; 5, Taenia spp. egg detected sample; 6, Strongyloides stercoralis larva detected sample; and 7, Trichuris trichiura egg detected sample.

PECT, the stool concentration technique, is a routinely used method for investigating parasitic infection. It has shown the highest sensitivity than the other microscope-based methods up to $100 \%$ in > 1000 eggs-per-gram (EPG) samples and exceeding $91 \%$ in cases of light infection [8]. As a result, we used PECT as a reference method for sensitivity, specificity, PPV, and NPV calculations. The best sensitivity from our study is of OvNad5 which showed $100 \%$ sensitivity followed by $O v N a d 2, O v N a d 4$, and OvNad1, respectively. Surprisingly, the $\mathrm{O} v \mathrm{Nad} 2$ and $\mathrm{O} v \mathrm{Nad} 5$ revealed better sensitivities than OvNad1 that was formerly established. OvNad5 showed the best efficiency in all investigated parameters including sensitivity, specificity, PPV, and NPV with $100 \%$. In comparison with the other target genes, OvNad5 also has better capacity than ITS-2 and Cox1 mentioned in previous reports by Lamaningao [25] and Buathong [15], with $83.1 \%$ and $89.1 \%$, respectively, especially in the light infection (less than 100 EPG) where the sensitivities of ITS-2 and Cox1 were up to $73.9 \%$ and $89.1 \%[10,15,23,24]$. DNA sequencing of the PCR products amplified by the introduced primers confirmed the sequences of $\mathrm{O} v \mathrm{Nad}$ that referred to the $\mathrm{Ov}$ eggs with less identity to the minute intestinal fluke. The introduced stool PCR, especially $\mathrm{O} v \mathrm{Nad}$, showed a relevant result to natural $O v$-positive samples investigated by PECT. All of the PECT-positive samples were also positive with OvNad5 PCR; interestingly, two PECT-negative samples were positive with $\mathrm{OvNad} 5 \mathrm{PCR}$, emphasizing the limitation of the conventional method to detect the egg in low-intensity samples. For the cross reactivities to other parasites that could be found together with $O v$ infection including Hookworm, $A$. lumbricoides, Taenia spp., S. stercoralis, E. vermicularis, and T. trichiura, the result suggested that OvNad has no cross reactivity with these parasite eggs. It is confidentially confirmed that OvNad subunits could be the exact targets for PCR amplification of $\mathrm{Ov}$-egg.
In conclusion, our study established that OvNad subunits can be the potential targets for $O v$-egg DNA detection in the stool specimen. Moreover, OvNad5 could be the best candidate due to the potent detection limit, highest sensitivity, specificity, PPV, and NPV. The introduced primers can be applied for using in routine diagnosis because conventional PCR is easier and cheaper than real-time PCR. It is also useful for other researchers to develop more tests on $\mathrm{O} v \mathrm{Nad}$ subunits in the future.

\section{Data Availability}

All data used to support the findings of this study are available from the corresponding author upon request.

\section{Ethical Approval}

The protocol was reviewed and approved by the Ethical Review Subcommittee Board for Human Research Involving Sciences of Thammasat University (Ref no. 153/2561), the Ethical Committee of the Faculty of Tropical Medicine, Mahidol University (Ref no. TMEC 20-2020), and the Ethics Committee of the Royal Thai Army Medical Department (Ref nos. S025 h/51 and S045 h/54).

\section{Conflicts of Interest}

The authors declare no conflicts of interest regarding the publication of this paper.

\section{Authors' Contributions}

WP, AGK, and RG conceived the research conceptualization. WP, SP, and PM conducted experiments, investigation, formal analysis, and statistical analyses. SP, KS, MM, TR, PA, and NL contributed specimens and materials. WP and PM 
wrote and finalized the manuscript. All authors reviewed and approved the final manuscript for submission.

\section{Acknowledgments}

This study was financially supported by a grant through the Faculty of Allied Health Sciences, Thammasat University, contract no. 1/2561 and 3/2561, and Thammasat University Research Unit in Parasitic Diseases.

\section{References}

[1] B. Sripa, S. Kaewkes, P. Sithithaworn et al., "Liver fluke induces cholangiocarcinoma," PLoS Medicine, vol. 4, no. 7, p. e201, 2007.

[2] P. Sithithaworn, R. H. Andrews, N. Van De et al., "The current status of opisthorchiasis and clonorchiasis in the Mekong Basin," Parasitology International, vol. 61, no. 1, pp. 10-16, 2012.

[3] A. Suwannatria, P. Saichua, and M. Haswell, "Epidemiology of Opisthorchis viverrini infection," Advances in Parasitology, vol. 101, pp. 41-67, 2018.

[4] M. M. Kirstein and A. Vogel, "Epidemiology and risk factors of cholangiocarcinoma," Visceral Medicine, vol. 32, no. 6, pp. 395-400, 2016.

[5] S. A. Khan, S. Tavolari, and G. Brandi, "Cholangiocarcinoma: epidemiology and risk factors," Liver International, vol. 39, no. Suppl.1, pp. 19-31, 2019.

[6] Y. Ustundag, Y. Bayraktar, and R. T. Poon, "Cholangiocarcinoma: a compact review of the literature," World Journal of Gastroenterology, vol. 14, no. 42, pp. 6458-6466, 2008.

[7] S. Sayasone, J. Utzinger, K. Akkhavong, and P. Odermatt, "Repeated stool sampling and use of multiple techniques enhance the sensitivity of helminth diagnosis: a cross-sectional survey in southern Lao People's Democratic Republic," Acta Tropica, vol. 141, pp. 315-321, 2015.

[8] L. Charoensuk, I. Subrungruang, M. Mungthin, S. Pinlaor, and P. Suwannahitatorn, "Comparison of stool examination techniques to detect Opisthorchis viverrini in low intensity infection," Acta Tropica, vol. 191, pp. 13-16, 2019.

[9] T. H. Le, N. Van De, D. Blair, P Sithithaworn, and D. P McManus, "Clonorchis sinensis and Opisthorchis viverrini: development of a mitochondrial-based multiplex PCR for their identification and discrimination," Experimental Parasitology, vol. 112, pp. 109-114, 2006.

[10] K. R. Umesha, S. Kumar, A. Parvathi et al., "Opisthorchis viverrini: detection by polymerase chain reaction (PCR) in human stool samples," Experimental Parasitology, vol. 120, pp. 353-6, 2008.

[11] A. Parvathi, K. R. Umesha, S. Kumar, P Sithithaworn, I Karunasagar, and I Karunasagar, "Development and evaluation of a polymerase chain reaction (PCR) assay for the detection of Opisthorchis viverrini in fish," Acta Tropica, vol. 107, pp. 13-6, 2008.

[12] N. Suksumek, K. Leelawat, S. Leelawat, B Russell, and U LekUthai, "TaqMan real-time PCR assay for specific detection of Opisthorchis viverrini DNA in Thai patients with hepatocellular carcinoma and cholangiocarcinoma," Experimental Parasitology, vol. 119, pp. 217-224, 2008.

[13] Y. Arimatsu, S. Kaewkes, T. Laha, S.-J. Hong, and B. Sripa, "Rapid detection of Opisthorchis viverrini copro-DNA using loop-mediated isothermal amplification (LAMP)," Parasitology International, vol. 61, no. 1, pp. 178-182, 2012.
[14] X. Q. Cai, H. Q. Yu, and R. Li, "Rapid detection and differentiation of clonorchis sinensis and opisthorchis viverrini using real-time pcr and high-resolution melting analysis," Scientific World Journal, vol. 2014, Article ID 893981, 6 pages, 2014.

[15] S. Buathong, S. Leelayoova, M. Mungthin et al., "Development and evaluation of PCR methods based on cytochrome $c$ oxidase subunit one (coxl) and $\mathrm{NADH}$ dehydrogenase subunit one gene (nad1) to detect Opisthorchis viverrini in human fecal samples," Parasitology Research, vol. 114, no. 9, pp. 3547-3549, 2015.

[16] R. Sahu, D. K. Biswal, B. Roy, and V. Tandon, "Molecular characterization ofOpisthorchis noverca(Digenea: opisthorchiidae) based on nuclear ribosomal ITS2 and mitochondrial COI genes," Journal of Helminthology, vol. 90, no. 5, pp. 607-614, 2016.

[17] S. Buathong, S. Leelayoova, M. Mungthin et al., "Molecular discrimination of Opisthorchis-like eggs from residents in a rural community of central Thailand," PLoS Neglected Tropical Diseases, vol. 11, no. 11, Article ID e0006030, 2017.

[18] S. Pumpa, W. Phadungsil, and R. Grams, "Improvement of a PCR-based method for the detection of Opisthorchis viverrini eggs in human stool samples by targeting internal transcribed spacer-2 (ITS-2), cytochrome oxidase subunit 1 (cox1), and cytochrome b (cyb)," Journal of Parasitic Diseases, vol. 45, pp. $474-478,2021$.

[19] C. A. Gordon, D. J. Gray, G. N. Gobert, and D. P. McManus, "DNA amplification approaches for the diagnosis of key parasitic helminth infections of humans," Molecular and Cellular Probes, vol. 25, no. 4, pp. 143-152, 2011.

[20] Y.-P. Xue, M.-C. Kao, and C.-Y. Lan, "Novel mitochondrial complex I-inhibiting peptides restrain NADH dehydrogenase activity," Scientific Reports, vol. 9, no. 1, p. 13694, 2019.

[21] S. J. Kerscher, "Diversity and origin of alternative NADH: ubiquinone oxidoreductases," Biochimica et Biophysica Acta (BBA) - Bioenergetics, vol. 1459, no. 2-3, pp. 274-283, 2000.

[22] S. Weinstein, N. A. Obuchowski, and M. L. Lieber, "Clinical evaluation of diagnostic tests," American Journal of Roentgenology, vol. 184, no. 1, pp. 14-19, 2005.

[23] K. Duenngai, P. Sithithaworn, U. K. Rudrappa et al., "Improvement of PCR for detection of Opisthorchis viverrini DNA in human stool samples," Journal of Clinical Microbiology, vol. 46, no. 1, pp. 366-368, 2008.

[24] M. Sato, U. Thaenkham, P. Dekumyoy, and J. Waikagul, "Discrimination of O. viverrini, C. sinensis, H. pumilio and $\mathrm{H}$. taichui using nuclear DNA-based PCR targeting ribosomal DNA ITS regions," Acta Tropica, vol. 109, no. 1, pp. 81-83, 2009.

[25] P. Lamaningao, S. Kanda, S. Laimanivong et al., "Development of a PCR assay for diagnosing trematode (Opisthorchis and haplorchis ) infections in human stools," The American Journal of Tropical Medicine and Hygiene, vol. 96, no. 1, pp. 221-228, 2017. 